\title{
Un aporte teórico-metodológico sobre la internacionalización de las políticas públicas*
}

A Theoretical-Methodological Contribution

on the Internationalization of Public Policies

\section{EMILIANO FERNÁNDEZ**}

Consejo Nacional de Investigaciones Científicas y Técnicas, Argentina.

Universidad Nacional del Centro de la Provincia de Buenos Aires, Argentina.

emilianofernandez33@gmail.com

https://doi.org/10.46468/rsaap.14.2.A7

El objetivo del presente trabajo es plantear una aproximación teóricometodológica al concepto de internacionalización de las políticas públicas. Desde una perspectiva marxista, y desde la recuperación crítica de aportes conceptuales del mainstream, se exponen y sintetizan los principales elementos teóricos que definen a este concepto y sus principales implicancias para el estudio concreto de las políticas públicas. En el marco de este objetivo, se presenta la internacionalización de las políticas públicas en el seno del proceso más amplio de internacionalización del Estado, para luego desarrollarlo en sus diferentes aspectos. El trabajo finaliza con la sistematización de los principales criterios teórico-metodológicos para el análisis de las políticas públicas que se desprenden de la propuesta teórica presentada.

\section{Introducción}

En términos generales, el campo de discusión de las políticas públicas está trazado por un fuerte nacionalismo metodológico (Hirsch y Kannankulam, 2010), que tiende a omitir o subestimar la dimensión internacional en el desarrollo de las mismas. Aun cuando se reconoce la presencia de algún elemento internacional en el desarrollo de una política pública en particular, se lo hace considerándolo exterior a la misma, con una incidencia más o menos mecánica sobre ella. Así, la dimensión internacional no tiende a reconocerse como constitutiva y constituyente del proceso de desarrollo de las políticas públicas.

* Artículo recibido el 4 de febrero de 2020 y aceptado para su publicación el 21 de septiembre de 2020.

** El autor agradece el aporte de dos evaluaciones anónimas a una versión previa de este artículo. 
Este trabajo busca discutir el problema de cómo interpretar la relación entre el desarrollo de las políticas públicas y los elementos que aparecen como exteriores al marco nacional. Es decir: ¿cómo interpretar la relación de las políticas públicas con los marcos normativos internacionales? ¿Cómo explicar la convergencia de políticas públicas a nivel internacional? ¿Cómo entender la relación de las políticas públicas con los lineamientos de los organismos internacionales o los espacios supranacionales?

Como vía para describir y explicar el conjunto de fenómenos que atraviesan los problemas antes señalados, proponemos la categoría de "internacionalización de las políticas públicas", contenida en una categoría más amplia como es la de "internacionalización del Estado" (Ciolli, 2018; Hirsch y Kannankulam, 2010; Picciotto, 1991). En este sentido, el objetivo de este trabajo será exponer los elementos que consideramos que definen la categoría teórico-metodológica de internacionalización de las políticas públicas y sus implicancias para el estudio de las mismas.

Nuestro planteo se asienta en una perspectiva marxista, aunque recuperaremos críticamente aportes provenientes del mainstream. La recuperación crítica incorpora elementos del plano de la caracterización de los fenómenos sin por ello asumir sus fundamentos teóricos (Adorno, 2001). Se trata, en particular, de las contribuciones de los estudios de difusión o transferencia de políticas públicas, que toman fuerza hacia la década del sesenta en el análisis de los Estados norteamericanos (Gray, 1973a; Gray, 1973b; Ross y Homer, 1976; Walker, 1969), y continúan hasta la actualidad en el seno de los estudios de políticas comparadas (Berry y Berry, 2010; Bulmer et al., 2007; Dolowitz y Marsh, 1996; Jakobi, 2009; Gilardi y Wasserfallen, 2019; Menseguer y Gilardi, 2008; Weyland, 2006; Weyland, 2011). ${ }^{1}$

Organizamos el trabajo en tres apartados. En el primer apartado, exponemos las determinaciones generales de la internacionalización del Estado, en el que se inscribe el proceso de internacionalización de las políticas públicas. ${ }^{2}$ En el segundo apartado, presentamos, en primer lugar, los elementos que hacen a la naturaleza de la internacionalización de las políticas pú-

En términos generales, desde el punto de vista teórico, estos estudios descansan en el pluralismo o bien en el institucionalismo político, ya sea desde un marco de elección racional limitada o no limitada. Por otro lado, también en términos generales, plantean el problema de la difusión como parte de un problema más amplio: la innovación o adopción de políticas. Es decir, la difusión de las políticas públicas se analiza en estos trabajos como un mecanismo (o fuente) más en la adopción las mismas. Por esto, tienden a circunscribir los análisis centralmente a la fase de toma de decisión o diseño de la política pública. Por último, como está planteado en los primeros estudios de la política norteamericana, la difusión es estudiada no solo entre estados nacionales, sino también entre estados subnacionales.

2 Utilizamos la noción de determinación en su sentido crítico dialéctico, en tanto: “(...) momento constitutivo esencial de la cosa” (Dussel, 1985:32). 
blicas (aquello que la internacionalización es, y por quienes está marcada); en segundo lugar, exponemos los elementos que configuran los canales y mecanismos de la internacionalización (los determinantes más específicos de la misma); y, en tercer lugar, planteamos los principales efectos de este proceso. En el cuarto apartado, analizamos las implicancias teóricometodológicas más importantes del marco de análisis trazado.

\section{La internacionalización de las políticas públicas como aspecto de la internacionalización del Estado}

En tanto las políticas públicas forman parte de la actividad estatal, explicar su internacionalización exige explicar antes la internacionalización del Estado y la relación nacional-global en las relaciones sociales capitalistas.

\section{1- La relación nacional-global \\ en las relaciones sociales capitalistas}

La especificidad de las relaciones sociales capitalistas es la separación objetiva entre la economía y la política, es decir, la separación entre el mercado y el Estado. De la forma capital, mediada por la libertad de quienes venden y quienes compran fuerza de trabajo, deriva la forma Estado. ${ }^{3}$ La forma mercado y la forma Estado son modos de existencia diferenciados de las mismas relaciones sociales capitalistas, constituyen una unidad en la separación (Bonnet, 2018; Hirch, 2017; Holloway y Picciotto, 1977). De este modo, la forma Estado está determinada por el antagonismo entre el capital y el trabajo. Además, en el Estado se expresa la condensación material y específica de relaciones de fuerza sociales, en las cuales se procesa un modo histórico de hegemonía (Block, 1987; Gramsci, 1984; Poulantzas, 2005).

El Estado es constituido por —y constituyente de- la unidad orgánica de lo nacional y lo internacional, en tanto dos momentos de la totalidad de

En la medida en que la libertad — la separación de los productores directos de los medios de producción - compele a los mismos a vender su fuerza de trabajo para conseguir sus medios de vida como única alternativa (coacción económica), no se requiere la fuerza física en el proceso inmediato de explotación (coacción extraeconómica). Al mismo tiempo, en la medida en que esta fuerza física niega la libertad, el otro aspecto de la libertad —el contrato de compra-venta libre de fuerza de trabajo - necesita, para cumplirse, que la misma se separe del proceso inmediato de explotación. Esta separación entre poder coactivo y proceso inmediato de producción es la forma distintiva y particularizada de la dominación capitalista (Holloway y Picciotto, 1977). 
las relaciones sociales capitalistas. Lo nacional y lo internacional no son dos entidades o esferas externamente relacionadas, sino partes de una misma totalidad -momentos de una misma unidad orgánica. Ni lógica ni históricamente existe un a priori en el que se conforman como entidades o ámbitos separadamente para luego entrar en un proceso de interrelación. Del mismo modo, lo global no puede plantearse como suma de naciones o de Estados-nación. Más bien, lo global y lo nacional son "una diferencia en la unidad" (Bonefeld, 2013), son modos de existencia diferenciados de las mismas relaciones sociales capitalistas. Los Estados-nación son la forma en la que se particularizan las relaciones sociales capitalistas globales en términos políticos y en la que se expresa la territorialización de esas relaciones en un espacio geográfico determinado. Así, lo global existe en esas particularizaciones nacionales —el sistema de Estados-, y al mismo tiempo lo nacional existe a través de esas relaciones globales. Al respecto, Bonefeld dice: "El Estado-nación existe a través de las relaciones globales de explotación y está confinado dentro de los límites impuestos por la forma contradictoria de la acumulación de capital a escala mundial" (2013:67).

Las relaciones internacionales, entonces, son el campo de fuerzas en que se resuelve la interacción entre los diversos Estados-nación, los cuales no son entidades de poder político in abstracto sino formas sociales de la totalidad global de las relaciones sociales capitalistas (Holloway, 1993). Las relaciones sociales capitalistas, aunque organizadas políticamente bajo la forma del Estado-nación, son inherentemente (y crecientemente) globales. El mercado mundial, el espacio donde se conforma la tasa media de ganancia que define la dinámica de los flujos de capital a través de los diferentes espacios nacionales de valorización, es la base de las relaciones internacionales entre esos Estados (Bonefeld, 2013). ${ }^{4}$ En este sentido, el mercado mundial y el sistema de Estados no son sino la forma económica y la forma política que respectivamente revisten las relaciones sociales capitalistas en el plano global. Por consiguiente, desde este punto de vista, la distinción entre lo nacional y lo global puede ser únicamente analítica, como disección metodológica sobre una unidad social real.

La forma social nacional —el Estado y el mercado doméstico- está determinada por la relación global de capital, y por su actividad en el siste-

\footnotetext{
"El mercado mundial no está constituido por muchas economías nacionales concentradas juntas, sino que el mercado mundial está organizado en la forma de muchas economías nacionales como sus componentes integrales. 'La primacía metodológica de la totalidad sobre los momentos singulares' también debe mantenerse en este nivel de la argumentación.” (Braunmühl, 2017:700). Y "El mercado mundial es un componente integral de esos procesos que tienen como resultado la afirmación del modo de producción capitalista y sus leyes. Esto significa que desde los orígenes del modo de producción capitalista el mercado mundial está integrado a las economías nacionales, en las cuales este proceso tiene lugar" (Braunmühl, 2017: 709 -710).
} 
ma interestatal y en el mercado mundial. Esta participación no es sino una forma derivada de ese primer momento global de la determinación, y por tanto está igualmente determinada por el antagonismo constitutivo de la relación de capital. Como argumentan Teschke y Lacher (2007), la forma de resolución de aquel antagonismo define la variable nacional e internacional de acumulación y territorialización del Estado en el marco de un sistema internacional que posee límites de coordinación y concertación. Pero también define aquella variable la competencia inter-capitalista en el mercado mundial (es decir, la competencia de capitales individuales por el reparto de plus-valor). De este modo, como afirma Pascual (2017), el Estado existe en una doble mediación: del antagonismo de clase en su territorio respecto del internacional (y viceversa) y de la competencia inter-capitalista en el mercado mundial. La competencia interestatal se define en esta doble mediación realizada por los Estados.

Por último, la dominación geopolítica de un Estado o alianza de Estados en un orden mundial cristaliza en instituciones, normas y mecanismos que establecen reglas generales de comportamiento para el conjunto de los Estados y para las fuerzas de la sociedad civil que operan por fuera de un ámbito nacional específico (Bieler y Morton, 2013; Burnham, 2013; Cox, 2014). Así, un orden mundial trazado por una determinada dominación geopolítica se materializa en instituciones que tienen como fin reproducirlo, expandirlo y galvanizarlo de las contradicciones internas.

\section{2- La internacionalización del Estado}

Entendemos que la internacionalización del Estado —al igual que la internacionalización del capital—es un aspecto del proceso de globalización intrínseca (y creciente) de las relaciones sociales capitalistas. Es decir, la internacionalización del Estado y la internacionalización del capital son, respectivamente, la forma política y la forma económica que asume la globalización constitutiva y permanente de las relaciones sociales capitalistas. ${ }^{5}$ Aunque la internacionalización del Estado es un proceso que se ubica en su propia matriz (pensemos, por ejemplo, en el constitucionalismo libe-

En las perspectivas marxistas, el problema de la internacionalización del Estado se plantea como parte de una problemática que concitaba (y tal vez aún concita) más interés: la internacionalización del capital. En efecto, la discusión sobre la internacionalización del Estado emerge en el seno del debate sobre las formas que asume la acumulación capitalista mundial desde la posguerra en adelante y, principalmente, desde la década de los setenta. El debate entre Warren y Murray (véase Picciotto, 1991) y el trabajo de Poulantzas (1973), son seguramente los principales antecedentes de este debate sobre el Estado desde el marxismo. 
ral decimonónico que cimentó el surgimiento de los Estados liberales), la misma se profundiza en las últimas cuatro décadas, de forma concomitante a la profundización de la internacionalización del capital.

La internacionalización del Estado es ante todo un proceso que descansa en un doble movimiento: la internalización (en un juego de inducción y adopción) de procesos y lineamientos político-institucionales internacionales en el Estado-nación; y, a su vez, la externalización de procesos y lineamientos político-institucionales desde el Estado-nación hacia el ámbito supranacional. El contenido último de este proceso de internacionalización del Estado, procesado en el doble movimiento de internalización-externalización, es la dominación nacional-global del capital sobre el trabajo. En su misma dinámica, se desarrollan las bases y condiciones para el proceso de reproducción (y de su contestación) del comando del capital sobre el trabajo a escala nacional y global. De aquí que nos parece acertada la afirmación de Picciotto (1991) sobre que, en última instancia, las contradicciones del proceso de internacionalización son las contradicciones congénitas del antagonismo del capital.

En este sentido, el movimiento de internalización de procesos y lineamientos político-institucionales que instituye el proceso de internacionalización del Estado aporta a la configuración las políticas públicas en el orden nacional y, a través de éstas, a la construcción de la forma particular que adoptan el Estado, el aparato estatal y sus funciones. Por otro lado, el movimiento de externalización del proceso de internacionalización configura el sistema interestatal, operando sobre los equilibrios geopolíticos del sistema de Estados. ${ }^{6}$ A ello se agrega, por otra parte, que este proceso de internacionalización no se sostiene sobre bases ecuánimes o simétricas, sino que, más bien, refleja la relación de fuerzas asimétrica presente en el sistema internacional. O sea, está determinado en gran medida por el predominio de los Estados centrales, y se convierte, en general, en un canal por el cual éstos buscan reproducir su dominación geopolítica.

Como parte del momento de externalización, la internacionalización implica la diversificación espacial de los espacios gubernamentales, adquiriendo importancia los niveles supranacionales (Brand y Wissen, 2010). A pesar de esta diversificación en escalas de las tomas de decisión política, los principales soportes de las mismas continúan siendo los Estados y, en parti-

\footnotetext{
En rigor, esto que indicamos para el movimiento de internalización y externalización en el proceso de internacionalización, también se puede producir a la inversa. Es decir, también la externalización de procesos y lineamientos político-institucionales modifica la forma particular del Estado y el modo de dominación a nivel interno como, a la inversa, la internalización modifica los equilibrios de fuerza hacia el interior del sistema de Estados. Arriba hicimos énfasis en el aspecto que nos parece más relevante.
} 
cular, los Estados capitalistas centrales. Esto, claro, no quiere decir que las instituciones internacionales no desarrollen dinámicas propias, pero ciertamente las mismas no transcienden los confines impuestos por los Estados miembros (Hirsch y Kannankulam, 2010).

La última fase de la internacionalización del Estado, la que corresponde a la ofensiva neoliberal, mediatizó e impulsó la agenda de reformas asociadas al neoliberalismo (Hirsch, 1999). Advertimos que no se trató de un proceso de extinción o de disminución del Estado, sino más bien de su metamorfosis (Bonnet, 2007; Oszlak, 2000; Picciotto, 2010). Descartamos, entonces, el planteo que tiende a identificar el incremento de la influencia de ciertas organizaciones internacionales —es decir, la intensificación de la internacionalización - con el debilitamiento del Estado. Más bien, la internacionalización tiende a fortalecer la capacidad del Estado de disciplinar a la clase trabajadora.

\section{La internacionalización de las políticas públicas}

Organizamos este apartado diseccionando el problema de la internacionalización de las políticas públicas, distinguiendo: la naturaleza del proceso de internacionalización de las políticas públicas, es decir, qué es en términos generales el proceso de internacionalización, y quiénes lo protagonizan; los fundamentos del proceso de internacionalización de las políticas públicas, es decir, cuáles son las determinaciones específicas que lo explican; los efectos del proceso de internacionalización de las políticas públicas, es decir, cuáles son los principales resultados en el desarrollo de éstas.

\section{1- Sobre la naturaleza del proceso \\ de internacionalización de las políticas públicas}

La internacionalización de las políticas públicas es un modo específico de internacionalización del Estado. En este sentido, la internacionalización de las políticas públicas y la internacionalización del Estado se constituyen recíproca y simultáneamente. La naturaleza del proceso de internacionalización de las políticas públicas reproduce, entonces, la naturaleza de la internacionalización del Estado. De esta manera, se asienta en el mismo doble movimiento: la internalización de lineamientos político-institucionales internacionales en el Estado-nacional y, a su vez, la externalización de lineamientos políticos-institucionales desde el Estado-nacional hacia el ámbito supranacional. Estos lineamientos están orientados al desarrollo de políticas 
públicas y remiten a ideas, normas, instrumentos o 'paradigmas de política“ (en términos de Hall, (1993): lineamiento que sostiene un marco de diagnóstico sobre un problema en particular, objetivos a alcanzar e instrumentos con los cuales llevar adelante los mismos).

El proceso de internacionalización de políticas públicas suele dar lugar a "olas" de expansión de determinado lineamiento político-institucional a través de distintos países. Al mismo tiempo, el despliegue temporal de estas olas, a menudo, es un proceso que comienza con lentitud, luego se acelera y finalmente vuelve a desacelerarse. Así, como resultado de la frecuencia acumulada, la secuencia de difusión sigue un patrón con forma de una curva en S. Por otra parte, este proceso de expansión de lineamientos frecuentemente posee como resultado procesos de convergencia de políticas públicas. De esta forma, políticas públicas de diferentes países con diversas características económicas y/o políticas adquieren similares patrones político-institucionales (Weyland, 2006). Por ejemplo, esta forma de internacionalización (en olas, de menor a mayor fuerza y tendiente a la convergencia) se puede encontrar en el área de la política social: como sucede con el paradigma bismarkiano de seguridad social de fines del siglo XIX, con los Fondos Sociales de Inversión de las dos últimas décadas del siglo XX, o con los más recientes Programas de Transferencias Monetarias Condicionadas (Bandeira, 2012; Franzoni y Voorend, 2011; Hickey y Seekings, 2017; Howlett et al., 2018; Osorio Gonnet, 2015, 2017; Sá y Silva, 2017).

Desde el punto de vista del momento de la internalización, la internacionalización interviene sobre el conjunto de procesos que articulan el desarrollo de las políticas públicas a escala local. En principio, puede intervenir en el propio proceso de construcción de la cuestión (Oszlak y O'Donnell, 2007), o sea, en la dinámica en que un asunto es problematizado públicamente a nivel nacional. Así, un conjunto de ideas, normas y experiencias del ámbito internacional son articuladas por los distintos agentes que participan en la construcción de la agenda pública local. Esta articulación de elementos internacionales puede utilizarse para legitimar o justificar la aplicación (o derogación) de determinada política pública, para vetar la aplicación de alguna otra, entre otras opciones. De este modo, la internacionalización puede partir desde la propia configuración de la agenda política, que envuelve el desarrollo de las políticas públicas. Un ejemplo es la incidencia, en las agendas nacionales de países latinoamericanos, del lineamiento estadounidense de militarización de los temas de seguridad interior y narcotráfico, que cuenta con el caso colombiano como referencia en la región (véase Tokatlian, 2004). Asimismo, la internacionalización también puede intervenir sobre las funciones de formulación (y reformulación), implementación y evaluación de las políticas públicas (Nakamura, 1987). 
Este punto es muy importante, porque da cuenta de la complejidad del proceso de internacionalización de las políticas públicas, en tanto un proceso que las permea como unidad. Un ejemplo de esto se observa en las características que reviste el proceso de asistencia técnico-financiera del Banco Mundial a la Asignación Universal por Hijo en la Argentina (2009-2020), donde se plasma un mecanismo de internacionalización específico como es el crédito internacional. Aquí, la asistencia técnico-financiera interviene en el rediseño del programa (plantea el pasaje de condicionalidades de educación y salud certificadas personalmente por los beneficiarios a un sistema automático realizado por las mismas dependencias estatales), en la implementación (estipula el aumento de la cobertura de sus beneficiarios), y también en la evaluación (impulsa el perfeccionamiento de los organismos estatales de evaluación de política social, además de la generación de una evaluación de impacto del programa) (Fernández, 2020).

Desde el punto de vista del momento de externalización del proceso de internacionalización, se observan como procesos centrales la configuración de la(s) agenda(s) internacional(es) y la construcción de lineamientos políticos internacionales. El proceso de formulación y desarrollo de una cuestión a nivel de las relaciones internacionales sigue lógica e históricamente a su desarrollo en los ámbitos nacionales. Esto quiere decir que la emergencia de cuestiones y el posterior establecimiento de una(s) agenda(s) internacional(es) parten del proceso de problematización y puesta en cuestión a nivel nacional. Así, una cuestión internacional tiene como punto de partida cuestiones nacionales. Esto no significa que la relación sea lineal y automática ya que, por el contrario, los modos de articulación de las agendas nacionales y globales poseen formas de expresión siempre sujetas a múltiples traducciones (y retraducciones). Además, es posible plantear la existencia de un ciclo vital de la cuestión internacional para delimitar y describir el proceso en que un asunto adquiere status de problema internacional, y es sujeto a múltiples posiciones, interpretaciones y estrategias de resolución. Asimismo, y como parte del mismo proceso de construcción del ciclo vital de la cuestión internacional, decanta un ciclo de lineamiento político internacional hacia las políticas públicas, en tanto período que engloba las estrategias dominantes de resolución (Oszlak y O’Donnell, 2007). Al igual que la agenda política nacional, la agenda política internacional clasifica y jerarquiza cuestiones, y delinea los contornos sobre qué cuestiones se deben tratar, cómo se deben hacer y cuándo. Aunque podríamos poner como ejemplo agendas internacionales sectoriales (la agenda nuclear, la ambiental, la de seguridad, entre otras.), planteamos el caso de una agenda internacional que centraliza y jerarquiza una gran cantidad de lineamientos internacionales sectoriales y constituye una fuerza ordenadora del proceso: la Agenda 2030 de las Naciones Unidas. Esta agenda, 
acordada en 2015 como continuación de los Objetivos de Desarrollo del Milenio 2000-2015, establece diecisiete Objetivos de Desarrollo Sostenible, entre los que se encuentran el fin de la pobreza, la educación de calidad, la igualdad de género, el agua limpia y el saneamiento, entre otros temas.

En cuanto a los lineamientos internacionales, que pueden ir de formas más flexibles (como ideas o principios de políticas) a formas más rígidas (como normas o paradigmas de políticas), se identifican diferentes modos de gestación. Esquemáticamente, marcamos dos generales. ${ }^{7}$ La primera modalidad está conformada por políticas públicas nacionales que en determinado momento se convierten en lineamiento internacional. De este modo, un caso nacional se transforma en referencia internacional, pudiendo ser incorporado a la orientación de organismos internacionales y/o expandiéndose de modo horizontal entre países. Se trata de una modalidad bottom up — de abajo hacia arriba. Existen muchos ejemplos, uno paradigmático es el caso de la privatización de los fondos previsionales de Chile (1981), que se tornó modelo de reforma previsional en todo el mundo. De 1981 a 2018 treinta países llevaron adelante algún tipo de privatización completa o parcial de sus sistemas previsionales: catorce en América Latina, catorce en Europa del Este y dos en África (Ortiz et al., 2018). Pero también encontramos el caso inverso, donde lineamientos internacionales se configuran en el propio ámbito internacional. Es decir, donde respuestas a cuestiones internacionales se construyen en organismos o espacios supranacionales, dando lugar a una modalidad top down — de arriba hacia abajo. Un ejemplo de esta modalidad lo encontramos en la idea de "trabajo decente" de la OIT (Organización Internacional del Trabajo) o, de modo más contundente, en su propuesta de "pisos de protección social", definidos como conjuntos de garantías básicas de seguridad social que deberían asegurar atención de salud esencial y seguridad básica en el ingreso que posibiliten el acceso a bienes y servicios básicos. ${ }^{8}$ Por último, sostenemos que los lineamientos internacionales pueden articularse de modo singular en distintos países, pero también pueden articularse en espacios supranacionales generando

Bulmer et al. (2007) identifican tres dimensiones de transferencias de políticas: downloading, que tiene lugar cuando las reglas de una espacio supranacional son aplicadas en los estados miembros (su ejemplo es la Unión Europea); uploading, que ocurre cuando un modelo de política de uno o algunos miembros de un espacio supranacional es incorporado por el mismo; y la dimensión horizontal, dada cuando las transferencias se basan en el aprendizaje reciproco entre estados miembros de un espacio supranacional.

8 Además, esta propuesta fue incluida, en abril de 2009, a las nueve iniciativas a ser promovidas por las Naciones Unidas. En el mismo año la CIT (Conferencia Internacional del Trabajo) aprobó el Pacto Mundial para el Empleo que incluye, además del concepto de Trabajo Decente, la necesidad de la protección social mediante un Piso de Protección Social para las denominadas "poblaciones vulnerables". 


\section{Emiliano Fernández}

marcos normativos y lógicas institucionales propias (Deacon, 2007; Deacon y Stubbs, 2013).

Ambos movimientos, de internalización de lineamientos internacionales (articulación de lo internacional a lo nacional) y de externalización (articulación de lo nacional a lo internacional) se constituyen recíprocamente. Están mutuamente mediados: la internalización media los elementos de la externalización (agenda y lineamientos internacionales) y la externalización media los elementos de la internalización (articulación a la agenda y políticas públicas nacional). La exteriorización existe en la mediación de la internalización y, viceversa, la internalización existe en la mediación de la externalización. Así, son momentos que constituyen una misma unidad, articulada temporal y escalarmente de modo diferencial.

Por otra parte, el proceso de internacionalización de políticas públicas es en gran medida anárquico, carece de un control central global, y en definitiva no está regido por la voluntad de ningún agente en particular (aunque haya agentes de mayor peso que otros en su definición). A pesar de que son varios los agentes que participan del proceso, planteamos tres principales: el personal de Estado (tanto administrativo como político), las organizaciones internacionales y la sociedad civil.

El personal de Estado (político y administrativo) participa de los procesos de internalización y externalización de los lineamientos internacionales como parte de su construcción de respuestas a los requerimientos de la acumulación y dominación en el espacio nacional. Las políticas públicas son esas respuestas estatales a dichos requerimientos. Las mismas están atravesadas por límites estructurales, los que remiten al propio modo de producción capitalista (como la propiedad privada de los medios de producción), pero también por límites internos que refieren a la racionalidad limitada del personal de Estado y a los problemas del aparato estatal para diagnosticar y responder a aquellos requerimientos (Álvarez y Bonnet, 2019; Lindblom, 1958). En este sentido, desde el punto de vista del personal de Estado, la incorporación de lineamientos internacionales se constituye en un insumo para los ajustes marginales (incrementales) de las políticas públicas. Pero también significan un insumo para responder a los momentos de crisis de las políticas públicas — de crisis de la inercia incremental—, y para la búsqueda de articulación de un nuevo paradigma de política (Hall, 1993). De este modo, la internacionalización puede envolver tanto los momentos de ajuste marginal de las políticas públicas como también los momentos de grandes cambios de política que responden a una crisis. Quizá el mejor ejemplo, en este punto, sea la crisis del paradigma keynesiano ahogado en distintos intentos de resolución, y la sustitución por el paradigma de política neoliberal vuelto lineamiento internacional. A caballo de la ofensiva del 
capital sobre el trabajo, este paradigma, impulsado por gobiernos y organizaciones internacionales, ofreció una respuesta a la crisis de la gestión keynesiana del capitalismo a nivel mundial (Babb, 2009 y 2013; Hall, 1993).

Los organismos internacionales también son agentes centrales en el proceso de internacionalización de las políticas públicas. Éstos revisten un papel de centralidad tanto en la expansión de lineamientos de política como en la construcción de la agenda internacional. Además, muchos actúan como como verdaderos "aparatos de hegemonía", retomando una expresión utilizada por Antonio Gramsci (1981) para caracterizar a la Iglesia Católica de su tiempo. En el interior de o asociados a estas organizaciones aparecen lo que denominamos núcleos de intelectuales orgánicos, en tanto grupos organizados para la función directiva y educativa (Gramsci, 1981). ${ }^{9}$ Estos núcleos tienen una función central en la elaboración, organización, difusión e instalación de lineamientos políticos internacionales que intervienen en el proceso de desarrollo de las políticas públicas. Se trata de una actividad de organización teórica y práctica de lineamientos que expresan supuestos teóricos, políticos y éticos. Desde esta unidad teórico-práctica, los núcleos intelectuales, entonces, se configuran como unidades de pensamiento e intervención con capacidad de operar en el campo teórico, político y técnico. En un sentido más específico, los núcleos intelectuales existen en diferentes formas: como grupos hacia el interior de grandes organizaciones (como los organismos de crédito), como think tanks, como consultorías, o como "redes de conocimiento" que atraviesan distintos espacios: público/privado, académico/político, nacional/internacional, entre otros. El terreno de los núcleos intelectuales es el terreno de la organización nacional/global de hegemonía de clase y, en menor medida, de la dominación geopolítica de Estados o alianzas de Estados.

Los diferentes agentes de la sociedad civil (ONG's, sindicatos, movimientos sociales, etcétera.) también participan del proceso de internacionalización de las políticas públicas, en la medida en que se vinculan con organizaciones internacionales y participan en el debate internacional o en el propio debate nacional a partir de orientaciones globales. Aquí entran tanto los agentes de la sociedad civil anclados localmente como los que poseen un marco de intervención específicamente internacional (por ejemplo, orga-

Utilizamos el concepto de intelectual orgánico desarrollado por Gramsci: “(...) los intelectuales tienen la función de organizar la hegemonía social de un grupo y su dominio estatal (...)" (1981: 188; C4, T2), y su planteo metodológico para entender a los intelectuales a partir de su función en las relaciones sociales, y no desde la naturaleza de su tarea. Entendemos que este concepto, en tanto se liga a la categoría de hegemonía y por tanto al fenómeno de la dominación política de clase, permite un análisis más complejo que el concepto que en general se suele utilizar para describir un fenómeno similar. Nos referimos al concepto de "comunidades epistémicas" (Adler y Haas, 2009; Deacon, 2007). 


\section{Emiliano Fernández}

nizaciones ambientales como Greenpeace, o de derechos humanos como Human Rights Watch, entre otras). Estos agentes muchas veces poseen vínculos directos con organizaciones internacionales o espacios supranacionales; no obstante, esto no sustituye ni quita centralidad al personal de Estado a la hora de centralizar, unificar y definir los procesos de desarrollo de la política pública en general.

\section{2- Canales de internacionalización de las políticas públicas}

Aunque el proceso de internacionalización es un fenómeno bien complejo, intentemos sistematizar los principales canales por el que se desenvuelve —competencia, coerción y aprendizaje—, y también los mecanismos centrales que el mismo proceso desarrolla (asistencia técnico-financiera, cooperación internacional y plataformas de difusión). ${ }^{10}$

El canal de internacionalización sostenido en la competencia (Berry y Berry, 2010; Weyland, 2011) remite a la presión emanada directamente del mercado mundial, que fuerza a los países a adoptar una u otra política en determinado momento. La presión competitiva sobre los Estados para captar o retener flujos de capital deriva, en general, en la adopción de medidas similares a las del país o espacio económico en que se compite (Clarke, 1989). Aunque ejemplos sobran, vale citar como referencia la reforma laboral emprendida por Schröder en Alemania (en el marco de la llamada Agenda 2010) a principios de siglo, que presionó por reformas laborales similares que bajaran costos laborales a sus pares de la Unión Europea (Clauwaert y Schömann, 2012).

El canal de internacionalización basado en la coerción (Dolowitz y Marsh, 1996; Menseguer y Gilardi, 2008) se sostiene sobre la presión externa de un organismo internacional o de un espacio supranacional, para aplicar determinada política como forma de sostener condiciones de financiamiento y/o la membresía ya sea del organismo o del espacio supranacional. El ejemplo típico, en el caso de la coerción derivada de organizaciones internacionales,

10 Los canales de internacionalización también pueden ser sistematizados en términos de un nivel horizontal (entre países) y en términos de una modalidad vertical (entre organizaciones o instituciones internacionales y países) (Jakobi, 2009); o bien entre canales voluntarios (aprendizaje) y canales con mayor grado de imposición (coerción o competencia). Por otra parte, es importante reconocer, con Menseguer y Gilardi (2008), que la clasificación de estos canales ("mecanismos de difusión" en la literatura especializada) coloca en simetría factores que no poseen el mismo valor explicativo: mientras que la coerción o la competencia explican de por si la internacionalización de una política, el aprendizaje no (más bien, la motivación del aprendizaje se encuentra en determinaciones «más internas» de la dinámica política y económica de un país). 
es el de las IFI's (Instituciones Financieras Internacionales). Tanto el Fondo Monetario Internacional (FMI) como el Banco Mundial cuentan con prolíficos ejemplos de programas financieros atados a condicionamientos. El reciente caso de la crisis griega es un botón de muestra del canal de coerción, donde la Troika -FMI, Banco Central Europeo y Comisión Europea-, en el marco de sucesivos "paquetes de rescate" (2010, 2012 y 2015), impuso lineamientos tales como: reforma laboral (eliminación de los convenios colectivos de trabajo, reducción del salario mínimo por debajo de los 600 dólares), reforma sanitaria (que incluyó recorte del gasto en salud y despido de enfermeros y médicos), reforma jubilatoria, privatización de servicios públicos, entre otras medidas (Bravo, 2018).

Por último, el canal de internacionalización basado en el aprendizaje descansa centralmente en la asimilación, por parte del personal de Estado, de lineamientos internacionales derivados de otras experiencias nacionales $o$ de recomendaciones y/o acuerdos con organismos internacionales y/o espacios supranacionales. ${ }^{11}$ A diferencia de los otros dos canales, no interviene la presión externa, sino que posee mayor impulso interno, y toma una forma más voluntaria. Un ejemplo de este canal puede hallarse en la difusión del paradigma de la Nueva Gestión Pública, donde la implementación de reformas administrativas (como nuevos sistemas de monitoreo y evaluación de resultados) en Chile y México estuvieron inspiradas en el aprendizaje de experiencias como la de Gran Bretaña (Hood, 1991); o en casos como la Argentina donde el aprendizaje estuvo (y está) más ligado a la asimilación de los lineamientos impulsados por el Banco Mundial o el Banco Interamericano de Desarrollo (López, 2005).

Por lo general, estos canales de internacionalización no se plasman de manera exclusiva, sino que se articulan de manera desigual y combinada. Así, por ejemplo, en la Unión Europea - una "plataforma de transferencia masiva de políticas" según Radaelli (2000)—, se dan procesos de difusión de políticas coercitivos (isomorfismo normativo), de aprendizaje (vía emulación) y también por competencia. Puede predominar un canal sobre otro, pero en general se dan de manera combinada. Esto, incluso, sucede con procesos de asistencia técnico-financiera de organismos internacionales, donde además de haber coerción también existen elementos de aprendizaje basados en acuerdos — de aquí que sea erróneo identificar créditos internacionales con el canal puramente coercitivo, como lo hace Osorio Gonnet (2015y 2017).

11 Entendemos al aprendizaje no desde la perspectiva racionalista (Menseguer y Gilardi, 2008), sino desde la perspectiva de la racionalidad limitada, sostenida en la teoría de las organizaciones (Simon, 1955) o, más recientemente, en la psicología cognitiva (Weyland, 2006). 
Estos canales de internacionalización están asociados de distinto modo a diferentes mecanismos de internacionalización. Los mecanismos de internacionalización pueden presentarse por separado, pero también de forma combinada.

El mecanismo de la cooperación internacional es llevado adelante por organizaciones internacionales o por países, y en general está orientado a países periféricos —entre estos mismos, se denomina "cooperación SurSur" En la modalidad tradicional de cooperación internacional, la misma aparece como "ayuda al desarrollo" de países centrales e instituciones internacionales a países atrasados y dependientes (Kern y Weisstaub, 2015). Bajo este mecanismo, los lineamientos político-técnicos de las instituciones internacionales se vehiculizan enmarcados en una estrategia que asume la forma de ayuda para el desarrollo. Este mecanismo no está exento de condicionalidades, corresponsabilidades y selectividades (Alonso y Sanahuja, 2006).

El segundo mecanismo es el financiero, concretado a través de créditos reembolsables a diferentes tasas de interés. Este mecanismo, obviamente, está estrechamente asociado a los organismos multilaterales de crédito. Éstos vierten dentro de su estrategia financiera un conjunto de lineamientos político-técnicos destinados a incidir en las políticas públicas en general (Babb, 2009 y 2013; Wade, 2001). Una de las principales distinciones entre este mecanismo y el primero es su lógica de rentabilidad financiera (aunque sus tasas de interés estén muy por debajo del mercado). El CAD (Comité de Ayuda para el Desarrollo) define a la Ayuda Oficial para el Desarrollo (ODA por sus siglas en inglés: Oficial Development Aid) a partir del porcentaje de donación que posee el otorgamiento de recursos y a partir de la tasa de interés. ${ }^{12}$

Ambos mecanismos poseen como dispositivo ordenador de su intervención al programa. El programa es la unidad de intervención de éstos, funciona como marco estructurador de objetivos político-técnicos a desarrollar en algún área de la política pública durante una duración de tiempo determinada, al tiempo que articula un conjunto de componentes que con-

12 Tradicionalmente la ODA contiene donaciones y préstamos realizados a un tipo de interés por debajo del mercado y con un elemento de donación del $25 \%$ como mínimo. Este último punto cambió en 2014. Según el tipo de país destinatario de la ayuda reembolsable, el elemento de donación exigido varía entre un $10 \%$ y un $45 \%$. En cuanto a la tasa de descuento de referencia, tradicionalmente se consideraba el $10 \%$ pero tras la reforma se sitúa entre el $6 \%$ y el $9 \%$ (nuevamente dependiendo del país socio). La reforma entra definitivamente en vigor en 2019 (para la contabilización de los flujos de 2018). Al respecto, véase el sitio web "Realidad de la Ayuda”, de la ONG Oxfam Intermón (representante de España de Oxfam Internacional): http://www.realidadayuda.org. 
tienen una serie de actividades a desarrollar por los distintos agentes involucrados. Los programas poseen un inicio, un desarrollo y un cierre delimitados a priori (que puede ser recalculado en el proceso de implementación), por lo que es válido hablar de ciclos de programas.

El tercer mecanismo lo podemos denominar "plataforma de difusión", Consiste en diferentes formas de divulgación de lineamientos internacionales: conferencias internacionales, foros internacionales, talleres internacionales de formación, visitas y viajes de estudio, entre otros dispositivos. Estas plataformas pueden ser desplegadas por países, por organismos internacionales o por espacios supranacionales, de manera exclusiva o asociada.

En el despliegue de un mecanismo en particular pueden convivir más de un canal de internacionalización, y viceversa, un canal de internacionalización puede articular más un de un mecanismo. Así, por ejemplo, en el primer caso, la puesta en juego del mecanismo del crédito, la asistencia técnico-financiera por parte de un organismo financiero, puede articular elementos coercitivos (desembolsos atados a determinados medidas administrativas), y al mismo tiempo elementos de aprendizaje (foros, seminarios o reuniones, donde personal de Estado interactúa con especialistas en algún campo de la política pública en particular). En el segundo caso, un canal como el del aprendizaje puede desarrollarse a partir de mecanismos como el de la cooperación internacional, y también a partir plataformas de difusión.

Asimismo, en los canales y mecanismos de internacionalización también participan otros factores, como, por ejemplo, la proximidad espacial o la semejanza de ciertas características económicas y culturales nacionales entre países.

\section{3- Efectos del proceso de internacionalización de las políticas públicas}

Desde el punto de vista del momento de la externalización del proceso de internacionalización, el conjunto del sistema político internacional puede ser pensado como producto histórico de dicho proceso. Las instituciones internacionales son resultado del proceso de internacionalización cristalizado en éstas, y al mismo tiempo condición del proceso de internacionalización en curso. Las instituciones internacionales (organismos internacionales y espacios supranacionales) le otorgan marcos políticonormativos, político-técnicos, teóricos, discursivos y financieros a los nuevos lineamientos internacionales emergentes. Y, desde el punto de vista del momento de internalización del proceso de internacionalización, desde las políticas públicas nacionales, el proceso de internacionalización tiene el efecto 
de aportar a su configuración (modificando, a su vez, el aparato estatal y sus funciones).

Al mismo tiempo, y como también se sugirió más arriba, se da un proceso de convergencia de políticas públicas a nivel internacional. Este fenómeno puede ser pensado como la expresión de una tendencia a la homogenización de las funciones estatales que se despliegan al interior de las mismas. A su vez, esta tendencia a la homogeneización como resultado del proceso de internacionalización política está en estrecha vinculación con el proceso de internacionalización del capital. Quizás este efecto de homogenización sea una de las principales implicancias del proceso de internacionalización del Estado y las políticas públicas. Por ejemplo, esta tendencia se observa patentemente en la expansión de las reglas de protección de inversiones contenidas en los tratados bilaterales de inversión (cláusulas de trato justo y equitativo, trato no discriminatorio, entre otras) y en las más nuevas reglas de facilitación de inversiones (que, desde el concepto de transparencia y previsibilidad, tienen el objetivo de simplificar administrativamente la circulación, instalación y salida de capitales a nivel nacional). Estas últimas reglas se inscriben en la llamada "cooperación reguladora" contenida en los tratados de comercio de segunda generación (TPP, TTIP y CETA ${ }^{13}$ ) o diversos acuerdos multilaterales de la OMC (Organización Mundial del Comercio), que exigen normas de cumplimiento obligatorio para los Estados firmantes (véase Ghiotto y Guamán, 2018). De esta forma, normativas y regulaciones nacionales muy diferentes entre sí, que rigen el desarrollo de políticas públicas en distintos países, tienden a homogeneizarse para facilitar el flujo de capital a nivel mundial. Otro ejemplo es el proceso que tiende a la homogenización de políticas públicas hacia la infraestructura física y logística para la inversión de capital dado en torno a la iniciativa IIRSA (Integración de la Infraestructura Regional Sudamericana), impulsada desde en el año 2000. Dicha iniciativa involucra la convergencia de políticas de doce países de la región (Argentina, Bolivia, Brasil, Chile, Colombia, Ecuador, Guyana, Paraguay, Perú, Surinam, Uruguay y Venezuela), y es apoyada por la asistencia técnica y financiera del BID (Banco Interamericano de Desarrollo), la CAF (Corporación Andina de Fomento) y el Fonplata (Fondo Financiero para el Desarrollo de la Cuenca del Plata) (véase Ciolli, 2020).

13 El Tratado Trans-Pacífico (TPP), el Tratado Transatlántico (TTIP) y el tratado entre Canadá y la Unión Europea (CETA). 


\section{Conclusiones}

En este trabajo buscamos exponer una propuesta para el análisis de las políticas públicas en la clave teórica-metodológica de la internacionalización. Nuestro punto de partida fue el carácter antagónico de las relaciones sociales capitalistas y sus modos de existencia diferenciados en el Estado y el mercado. En este marco, definimos a la internacionalización del Estado como el proceso de internalización y de exteriorización de lineamientos y procesos internacionales que contribuyen a configurarlo. Dicha internalización del Estado se produce a través de la propia internacionalización de las políticas públicas. Las mismas son respuestas estatales a los desafíos impuestos por la acumulación y la dominación, ambas mediadas por el antagonismo entre el capital y el trabajo. Dijimos, a su vez, que el proceso de internacionalización aporta a la forma en que las políticas públicas buscan responder dichos requerimientos; en este sentido, planteamos que dicha contribución puede enmarcarse en los pequeños ajustes marginales de las políticas públicas, o en los grandes giros de las mismas. Además, sostuvimos que puede procesarse, de forma exclusiva o combinada, por algunos canales: ya sea por la competencia que fuerza a implementar determinadas medidas estatales para retener o atraer flujos de capital; por la coerción donde un lineamiento es impuesto al Estado por parte de una institución internacional; y por aprendizaje donde existe un proceso de lecciones aprendidas por parte del personal de Estado. En el seno de esos canales se articulan mecanismos, en general de forma combinada, que viabilizan dicho proceso: la asistencia técnico-financiera, la cooperación internacional y las plataformas de difusión.

Más allá de que en el trabajo expusimos ejemplos históricos para ilustrar algunos de nuestros argumentos teóricos, y también para referir a las consecuencias histórico-concretas del proceso de internacionalización de las políticas públicas, nos interesa resaltar en este último punto la propuesta teórico-metodológica. O sea, las premisas teórico-metodológicas que creemos importantes para analizar políticas públicas sectoriales de un modo que supere los sesgos del nacionalismo metodológico. En este sentido, intentando sistematizar estas premisas, planteamos la necesidad de situar el análisis concreto de una política pública en particular: i) mediada por el proceso de internacionalización, por el movimiento de internalización y externalización de lineamientos internacionales; ii) inscripta en determinado orden mundial, con una particular configuración económica y geopolítica; iii) conectada con determinada agenda política internacional y sus lineamientos políticos; iv) vinculada a organizaciones internacionales, espacios supranacionales u organizaciones de la sociedad civil globales; v) 


\section{Emiliano Fernández}

expuesta a canales de internacionalización: competencia, coerción y/o aprendizaje; vi) articulada con mecanismos de internacionalización: asistencia técnico-financiera, cooperación internacional y/o plataformas de difusión; vii) elementos que inciden en la forma que adopta, el aparato que la gestiona y sus funciones y, en definitiva, en su capacidad para responder a los desafíos que impone la acumulación y la dominación a nivel nacional y viii) inscripta, como resultado de este proceso, en determinada tendencia de convergencia de políticas públicas a nivel internacional.

\section{Referencias bibliográficas}

Adler, E. y Haas, P. (2009). Las comunidades epistémicas, el orden mundial y la creación de un programa de investigación reflectivo, Relaciones Internacionales, 12, 149-169.

Adorno, T. (2001). "Sociología e investigación empírica”. En P. Torres, S. Sevilla y J. Talens Epistemología y ciencias sociales (pp.9-19). Madrid: Frónesis Cátedra Universitat de València.

Álvarez Huwiler, L y Bonnet, A. (2019). "Aportes para una crítica marxista de las políticas púbicas”. En Mallardi y Fernández (Comps.) Cuestión Social y políticas sociales (pp.107-137). Tandil: Ed. Puka.

Alonso, J. y Sanahuja, J. (2006). "Un mundo en transformación: repensar la agenda de desarrollo”. En Arias, M. (Comp.) La realidad de la ayuda 2006-2007 (pp. 179204). Barcelona: Intermón Oxfam.

Babb, S. (2009). Behind the development banks. Washington politics, world poverty, and the wealth of nations, London: The University of Chicago Press.

Babb, S. (2013). The Washington Consensus as transnational policy paradigm: Its origins, trajectory and likely successor. Review of International Political Economy, 20, 268-297.

Bandeira, D. (2012). Political Competition and the Diffusion of Conditional Cash Transfers in Brazil. Brazilian Political Science Review, 6(2), 56-87.

Berry, F. y Berry, W. (2010). "Modelos de innovación y difusión en el estudio de las políticas públicas”. En Sabatier, P. (Ed.) Teorías del proceso de las políticas públicas. Buenos Aires: Proyecto de Modernización, Jefatura de Gabinete.

Bieler, A. \& Morton A. (2013). "Hegemonía, orden mundial y cambio histórico: siguiendo el camino de la teoría crítica. Perspectivas neogramscianas en las relaciones internacionales”. En Kan J. y Pascual R. (Comps.), Integrados (?) Debates sobre las relaciones internacionales y la integración regional latinoamericana y europea. Buenos Aires: Imago Mundi.

Block, F. (1987). Revising State Theory. Essays in Politics and Postindustrialim. Philadelphia: Temple University Press, 
Bonefeld, W. (2013). "Más allá de las relaciones internacionales: acerca del mercado mundial y el Estado-nación”. En Kan J. y Pascual R. (Comps.) Integrados (?) Debates sobre las relaciones internacionales y la integración regional latinoamericana y europea. Buenos Aires: Imago Mundi.

Bonnet, A. (2007). La hegemonía menemista. El neoconservadurismo en Argentina, 1989-2001. Buenos Aires: Prometo Libros.

Bonnet, A. (2018) ¿Qué es el Estado capitalista? La derivación revisitada. En prensa. Brand, U. y Wissen, M. (2010). Approaching the Internationalization of the State: An Introduction. Antipode, 43, 1-11.

Braunmühl, C. (2017). "El análisis del Estado nacional burgués en el contexto del mercado mundial. Un intento por desarrollar una aproximación metodológica y teórica”. En Bonnet, A. y Piva, A. (Comps. y Eds.) Estado y Capital. El debate alemán sobre la derivación del Estado. Buenos Aires: Ediciones Herramienta.

Bravo, G. (23 de mayo de 2018) El FMI y el 'rescate' griego: más deuda y reformas antiobreras. La Izquierda Diario. Recuperado de: http://www.laizquierdadiario.com/ El-FMI-y-el-rescate-griego-mas-deuda-y-reformas-antiobreras

Bulmer, S., Dolowitz, D., Humphreys, P. y Padgett, S (2007) Policy Transfers in European Union Governance. Regulating the utilities. New York: Taylor y Francis.

Burnham, P. (2013). "Hegemonía neogramsciana y el orden internacional”. En Kan J. y Pascual R. (Comps.) Integrados (?) Debates sobre las relaciones internacionales y la integración regional latinoamericana y europea. Buenos Aires: Imago Mundi.

Ciolli, V. (2018). Burocracia estatal: entre la internacionalización y la territorialidad. Revista Relaciones Internacionales, 38, 79-98.

Ciolli, V. (2020). Notas para pensar la internacionalización del Estado a partir de su vínculo con los organismos financieros internacionales. En Ciolli, V., Naspleda, F. y Bernado, R. (Comps.) La dimensión inevitable: estudios sobre la internacionalización del Estado y del capital desde Argentina. Quilmes: UNQ.

Clarke, S. (1989). The Internationalisation of Capital and the Nation State. International Conference on 'New Forms of the Internationalisation of Capital'. Presentado en Conferencia en Washington, junio. Recuperado de https://homepages. warwick.ac.uk/ syrbe/pubs/washi.pdf.

Clauwaert, S. y Schömann, I. (2012). La crisis y las reformas de las legislaciones laborales nacionales en Europa. Un ejercicio de análisis. Anuario de Relaciones Laborales en España, 4, 67-70.

Cox, R. (2014) Fuerzas sociales, Estados y órdenes mundiales: Más allá de la Teoría de Relaciones Internacionales. Relaciones Internacionales, 24, 99-116.

Deacon, B. (2007). Global Social Policy and Governance. SAGE Publications Ltd.

Deacon B. y Stubbs P. (2013). Global social policy studies: Conceptual and analytical reflections. Global Social Policy, 13, 5-23.

Dolowitz, D. y Marsh, D. (1996). Who Learns What from Whom: a Review of the Policy Transfer Literature. Political Studies, XLIV, 343-357. 
Dussel, E. (1985). La producción teórica de Marx. Un comentario a los Grundrisse. México: Siglo XXI Editores.

Fernández, E. (2020). La internacionalización de la política asistencial argentina: El ciclo de asistencia técnico-financiera del Banco Mundial a la Asignación Universal por Hijo (2009-2018). En prensa.

Franzoni y Voorend (2011). Actors and ideas behind CCTs in Chile, Costa Rica and El Salvador. Global Social Policy, 11(2), 279-298.

Gilardi, F. y Wasserfallen, F. (2019). The politics of policy difussion. European Journal of Political Research, 58(4), 1245-1256.

Ghiotto, L. y Guamán, A. (2018). ¿Facilitación o protección para las inversiones extranjeras? Nuevos elementos del debate global. Ciclos, XXV(51), 1-18.

Gramsci, A. (1981). Los Cuadernos de la Cárcel, Tomo II. México: Ediciones Era.

Gramsci, A. (1984). Los Cuadernos de la Cárcel, Tomo III. México: Ediciones Era.

Gray, V. (1973a). Innovation in the States: A Diffusion Study. The American Political Science Review, 67(4), 1174-1185.

Gray, V. (1973b). Rejoinder to «Comment» by Jack L. Walker. The American Political Science Review, 67(4), 192-1193.

Hall, P. (1993). Policy Paradigms, Social Learning, and the State. The Case of Economic Policy making in Britain. Comparative Politics, 25 (3).

Hickey, S. y Seekings, J. (2017). The global politics of social protection. WIDER Working Paper, 2017, 1-29.

Hirsch, J. (2017). "El aparato de Estado y la reproducción social: elementos de una teoría del Estado burgués”. En Bonnet, A. y Piva, A. (Comps. y Eds.) Estado y Capital. El debate alemán sobre la derivación del Estado. Buenos Aires: Ediciones Herramienta. Libro Digital.

Hirsch, J. (1999). Globalización del capital y la transformación de los sistemas de Estado: del "Estado de seguridad" al "Estado nacional competitivo". Cuadernos Sur, 28, Año 15.

Hirsch, J. y Kannankulam, J. (2010). The Spaces of Capital: The Political Form of Capitalism and the Internationalization of the State. Antipode, 3, 12-37.

Holloway, J.y Picciotto, S. (1977). “Capital, crisis and State”. En Clarke, S. (Ed.) The State Debate. Capital and Class. London: Palgrave Mcmillan.

Holloway, J. (1993). La reforma del Estado: Capital global y Estado Nacional. Perfiles Latinoamericanos, 1, 7-32.

Hood, C. (1991). A Public Management for All Seasons? Public Administration, 69(1), 3-19.

Howlett, et al. (2018). Difusão dos PTCs da América Latina para a Ásia: o caso do programa 4Ps nas Filipinas, Revista de Administracao Pública, 54, 264-284.

Jakobi, A. (2009). International organizations and world society: studying global policy development in public policy. TranState Working Paper, 81.

Kern, A. y Weisstaub, L. (2015). Una nueva agenda mundial de desarrollo. Foreign Affairs, 15(3), 139-145. 
Nakamura, R. (1987). The textbook policy process and implementation research. Policy Studies Review, 7(1), 142-154.

Menseguer, C. y Gilardi, F. (2008). Reflexiones sobre el debate de la difusión de políticas. En Política y gobierno, XV(2), 314-351.

Lindblom, C. (1958). Policy Analysis. The American Economic Review, 48 (3), 298-312.

López, A. (2005). "Los fundamentos de la Nueva Gestión Pública: lógica privada y poder tecnocrático en el Estado mínimo”. En Thwaites Rey, M. y López, A. (Comps.) Entre tecnócratas globalizados y políticos clientelistas. Derrotero del ajuste neoliberal en el Estado argentino. Buenos Aires: Prometo.

Ortiz, I., Durán-Valverde, F., Urban, S. y Wodsak V. (2018). Reversing Pension Privatizations. Rebuilding public pension systems in Eastern Europe and Latin America. Ginebra: International Labour Office.

Osorio Gonnet, C. (2015). Mecanismos de difusión de los Programas de Transferencia Condicionada en América Latina. El caso chileno. Iconos, 53, 31-48.

Osorio Gonnet, C. (2017). ¿Cómo viajan las ideas? El rol de las comunidades epistémicas en el diseño de políticas sociales en América Latina. Revista CLAD, Reforma y Democracia, 68, 77-112.

Oszlak, O. (2000). El mito del Estado mínimo: una década de reforma estatal en Argentina”. Trabajo presentado en el V Congreso Internacional del CLAD sobre Reforma del Estado y de la Administración Pública, Rep. Dominicana.

Oszlak, O. y O‘Donnell, G. (2007). "Estado y políticas estatales en América Latina: hacia una estrategia de investigación2. En Acuña, C. (Comp.) Lecturas sobre el Estado y las políticas públicas: Retomando el debate de ayer para fortalecer el actual. Buenos Aires: Jefatura de Gabinete de Ministros de la Nación.

Pascual, R. (2017). Conflicto/cooperación, intergubernamentalidad/upranaciona- lidad: antagonismo social de clase y relaciones internacionales. Ponencia presentada en las jornadas "25 años de marxismo abierto”, Universidad de Puebla, México.

Picciotto, S. (1991). "The internationalisation of Capital and the International State System”. En Clarke, S. (Ed.) The State Debate. Senior Lecturer in Sociology. Reino Unido: University of Warwick.

Picciotto, S. (2010). International Transformations of the Capitalist State. Antipode, 43, 87-107.

Poulantzas, N. (1973). La internacionalización de las relaciones capitalistas y el Estado nación. Investigación Económica, 31(127).

Poulantzas, N. (2005). Poder político y clases sociales en el Estado capitalista. México: Siglo XXI.

Radaelli, C. (2000). Policy Transfer in the European Union; Institutional Isomorphism as a Source of Legitimacy. Governance, 13(1): 25-43.

Ross, M. y Homer, E. (1976) Galton's Problem in Cross-National Research. World Politics, 29, 1-28.

Sá e Silva, M. (2017). Poverty Reduction, Education, and the Global Diffusion of Conditional Cash Transfers. Suiza: Palgrave Mcmillan. 
Simon, H. (1955). A Behavioral Model of Rational Choice. The Quarterly Journal of Economics, 69(1).

Teschke, B. y Lacher, H. (2007). The changing 'logics' of capitalist competition. Cambridge Review of International Affairs, 20, 565-580.

Tokatlian, G. (2004). "Colombia: más inseguridad humana, menos seguridad regional”. En Gómez, J. (Comp.) América Latina y el (des) orden global neoliberal. Buenos Aires: CLACSO.

Wade, R. (2001). Showdown at the World Bank. New Left Review 7, 124-137.

Walker, J. (1969). The diffusion of Innovations among the American States. The American Political Science Review, 63, (3), 880-899.

Weyland, K. (2006). Bouded rationality and policy diffusion. Social Sector Reform in Latin American. Nueva Jersey: Princeton University Press.

Weyland, K. (2011). Cambio institucional en América Latina: modelos externos y consecuencias no previstas. América Latina Hoy, 57, 117-143.

Palabras clave:

Políticas Públicas - Internacionalización - Estado - Capital - Instituciones Internacionales

\title{
Key words
}

Public policy - Internationalization - State - Capital - International institutions

\begin{abstract}
Abtract
The objective of this paper is to propose a theoretical-methodological approach to the concept of internationalization of public policies. From a marxist perspective, and from the critical recovery of conceptual contributions from the mainstream, the main theoretical elements that define this concept and its main implications for the concrete study of public policies are exposed and synthesized. Based on this objective, the proposal of the internationalization of public policies is presented within the broader process of internationalization of the state, and then developed in its different aspects. The work ends with the systematization of the main theoreticalmethodological criteria for the analysis of public policies that emerge from the theoretical proposal presented.
\end{abstract}

